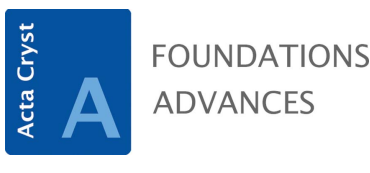

ISSN 2053-2733

\section{A Journey into Reciprocal Space: A Crystallographer's Perspective. By A. M. Glazer. Morgan \& Claypool, 2017. Paperback, pp. 190. Price USD 55.00. ISBN 9781681746203.}

\author{
Berthold Stöger*
}

Technische Universität Wien, X-ray Centre, Getreidemarkt 9, 1060 Wien, Austria. ${ }^{*}$ Correspondence e-mail: bstoeger@mail.tuwien.ac.at

For students of crystallography and solid-state physics, coming to terms with reciprocal space means travelling down a thorny road. In this context, a book titled $A$ Journey into Reciprocal Space: A Crystallographer's Perspective by A. M. Glazer, published by IOP Publishing in the 'Concise Physics' series, could fill a glaring gap. The target audience is solid-state physicists who need a gentle introduction to crystallographic concepts and an overview of the field as seen through the eyes of a crystallographer. The book can be divided into a part on crystallography (Chapters 1-3) and a part on solid-state physics (Chapters 5 and 6), with Chapter 4 ('Dynamical diffraction') bridging the two. The book has numerous positive aspects. For example, the author warns of common pitfalls (for example monoclinic meaning $\beta \neq 90^{\circ}$ ), denounces misconceptions such as 'lattice structure' and recounts interesting historical anecdotes such as the 'photosommateur'. Practical applications (for example the metric tensor, measurement methods, Ewald construction, analysis of the Patterson map) are easy to follow.

Sadly, however, the presentation is not as rigorous as one could have expected. The less serious, but not less fastidious, problem is inconsistent typography, which gives a sloppy impression. Lengths, vectors and axes are typeset arbitrarily in bold and italics; differentials of volume integrals are systematically written as $\mathrm{dr}^{3}$ instead of the correct $\mathrm{d}^{3} \mathbf{r}$. F is designated an 'amplitude' and in the next line used as a complex number.

The parts of the book that contain the most errors are the mathematical derivations. Only two of the worst errors (one in each part) shall be mentioned here. The Fourier transform of a lattice is given as

$$
A(\mathbf{Q})=\int_{\text {lattice }} \exp (\mathrm{ir} \cdot \mathbf{Q}) \mathrm{dr}^{3},
$$

which is a very unfortunate notation, since it can be read as an integral over a set of points, in which case it will evaluate to 0 or perhaps as the Fourier transform of a constant-valued field, but hardly that of a lattice (is there a sum of $\delta$ distributions missing?). The result of the deduction appears deus ex machina: $A(\mathbf{Q})$ has only 'values at discrete points $h k l$ ', as if 0 were not considered a 'value'; the reader does not learn what the 'value' at ' $h k l$ ' is (as the given series tends to $+\infty$ ). The observed 'amplitude' is $\mathbf{F}(h k l)$ $=A(\mathbf{Q})$ (original typography), which is therefore infinity.

The derivation of Bloch's theorem is based on 'an operator $\hat{T}$ acting on the function $\exp (\mathbf{i k} \cdot \mathbf{r})$ ', but apparently also acting on vectors and/or scalars:

$$
\hat{T} \exp (\mathrm{ik} \cdot \mathbf{r})=\exp (\mathrm{i} \hat{T} \mathbf{k} \cdot \mathbf{r}) .
$$

Later it is stated that $\hat{T} \mathbf{p} \cdot \hat{T} \mathbf{q}=\mathbf{p} \cdot \mathbf{q}$. By setting $\mathbf{p}=\mathbf{q}$ we note that $\hat{T}$ cannot change the length of a vector just to be informed a few lines down that $\hat{T} \mathbf{r}=\mathbf{r}+\mathbf{t}_{n}$, which means that $\hat{T}$ does change the length. From these contradictions follows that $' \hat{T} \exp (\mathbf{i k} \cdot \mathbf{r})=[\ldots]=\exp (\mathbf{i k} \cdot \mathbf{r})$ ' and therefore 'suitable basis functions for translation can be taken as $\exp (\mathbf{i k} \cdot \mathbf{r})$ '. The meaning is unclear.

Sometimes, excess pedagogical effort becomes a trap, as in the example of the atomic form factor. We read (page 3-30) that it 'will drop off with angle faster for heavier, and thus larger, atoms', thus proving: '[l]arge distances [in real space] become small [in reciprocal space]'. As a matter of fact, the opposite is true: heavy elements scatter to 
higher angles owing to their tightness: the core electrons are closer to the nucleus because of the increased attractive force. The limit of the distribution of core electrons for a hypothetical atom of infinite weight is a Dirac delta, whose Fourier transform is a constant: the scattering power of such an atom would not drop off at all. Fig. 3.32 actually proves this: the light $\mathrm{O}$ atom retains only $20 \%$ but the heavier $\mathrm{K}$ atom $40 \%$ of its scattering power at high angles; this would be even more evident had the curves been normalized with respect to the number of core electrons. The author is apparently thinking in absolute terms, which makes the curve for $\mathrm{K}$ steeper overall. However, this is because of its higher total scattering power. Indeed, considering the normalized scattering power, 'larger atoms' in direct space also means a 'larger' contribution in reciprocal space, the opposite of what the author intended to say.

Terminological confusion is quite common among nonspecialists. This book, aimed precisely at non-specialists, should give a clear picture of concepts that are too often used inconsistently. Unfortunately, a certain fear of going into too much detail produced the opposite result, i.e. promoting the confusion one wanted to avoid. There is confusion between symmetry operations, geometric and symmetry elements; between point groups, point-group types and crystallographic point groups; between lattice system and crystal system [leading to the confusing section 'Hexagonal, trigonal (and rhombohedral)']. The 'element' of a higher rotoinversion (i.e. with a rotational component with order higher than 2) is given as a point, when of course a line is also needed to fully define its orientation. The book is often vague or inconsistent in other topics as well. For example, quartz 'exists in two crystalline habits that are mirror images of each other' (enantiomorphic is one habit) and then 'examples of crystal habit[s]' are given as 'needle-like' or 'prismatic' (but what would the 'mirror image' of 'needle-like' be?). A reader familiar with these terms may make sense of this, but a newcomer will end up very confused. Point symmetry is defined via 'operators', which are not defined, but apparently used here in the sense of a linear map from a vector space onto itself, "that describe symmetry operations that act through a point in space' (a 'symmetry operation' may leave points unchanged - it does not 'act through' them). 'Translation symmetry is the symmetry that is exhibited by a collection of equivalent objects repeated regularly throughout space' may be an explanation but is not a helpful 'definition', since such a 'collection of equivalent objects' may have other symmetries besides pure translations. 'Bravais lattices' are defined as 'unique types' without stating what the 'type' of a lattice is. It is not clear how a 'unit cell ... shows the true symmetry of a lattice', as the true symmetry of a lattice certainly involves translation, in contrast to a unit cell; the point group of the lattice is obviously meant here. A crystal is 'a solid' with 'an ordered arrangement in space'. Firstly, order is a more general and vague concept than crystallinity. The former is therefore not useful to define the latter. Secondly, most readers will be able to cope with the facts: Crystallinity used to be defined via translation symmetry but that was changed to a more phenomenological, perhaps not entirely satisfying, definition involving diffraction patterns. '[P]oint symmetry operators' are classified into the three types 'proper rotations, inversions and rotoinversions'. The identity (1) is discussed with 'proper rotations' $(2,3, \ldots)$ but the inversion (1) not with 'rotoinversions' $(\overline{3}, \overline{4}, \ldots)$. 'Reflections' (formally $\overline{2}$ ) are discussed in the section 'inversions' not 'rotoinversions'. This all appears rather arbitrary.

Some figures are confusing: In the Ewald construction of a Renninger reflection, the indicated reflection is $\mathbf{k}_{2}-\mathbf{k}_{1}$, but the label and main text state $\mathbf{k}_{1}-\mathbf{k}_{2}$; arrows are in real space but labelled with reciprocal-space vectors and the Renninger reflection is not located on the original Ewald sphere. The segments of the band structure in 'Nearly free electrons' all have the same slope, making it unclear that they are derived from a single parabola.

A slide-projector analogy to explain the 'phase problem' fails in multiple ways. It says that the 'purpose of the lens [of the projector] is to combine all the amplitudes and phases of the light wave from the object', when the phase plays no role in slide projection, as there is no interference involved. Moreover, for '[X]-ray and neutron diffraction' it is only 'the intensity [not the phase] that one observes ... because no lens is available', when again these are unrelated issues. Also, 'the light arriving at the screen contains all information about the slide at every place within the lit area' (emphasis added) might give the wrong impression that every point in reciprocal space contains the whole information if there was only an appropriate 'lens', which is contradicted by 'one needs the amplitudes and the phases of each $h k l$ reflection'.

In some cases crucial information is omitted: The trigonometrical proof (pages 1-20 and 1-21) of the crystallographic restriction theorem (not named as such) lacks the fundamental statement that $\mathbf{t}$ is the shortest lattice vector in a given direction, by confusing sin and cos (equations 1.16 and 1.17 should contain the cosine, not the sine) and by omitting the -1 for $\sin \alpha$ in the list of its possible values. The Laue equation is derived from the 'path difference' of waves scattered at only two neighbours of a 'row of atoms', which is insufficient to explain sharp diffraction peaks: the 'path difference' of waves scattered by atoms farther apart would have to be taken into account. The periodicity of ' $u_{k}$ ' in ' $u_{k} \exp (\mathbf{i k} \cdot \mathbf{r})$ ' is only given indirectly by writing it as a discrete Fourier series (without naming it as such). The displacement of the 'sth atom' in the classical mass-and-spring model does not depend on $s$. To prove periodicity of $k$-space the reader should consider a 'plane wave' and 'subtract a reciprocal lattice vector $\mathbf{g}$ from the wavevector':

$$
\exp \left[\mathrm{i}(\mathbf{k}-\mathbf{g}) \cdot \mathbf{t}_{n}\right]=\exp \left(\mathbf{i k} \cdot \mathbf{t}_{n}\right) \exp \left(-\mathrm{ig} \cdot \mathbf{t}_{n}\right),
$$

where $\mathbf{t}_{n}$ is a (direct) lattice vector. These are constant complex numbers, not plane waves (which would need a position vector). How this proves that 'the wave solutions are invariant under addition or subtraction of $[\ldots] \mathbf{g}$ ' is unclear.

Though not wrong, some derivations could have been shortened significantly if the proper concepts had been used, and thus been rendered more insightful. For example, the 
derivation of the Patterson function would become trivial by application of the convolution theorem (which is used in other parts of the book). The phase problem could have been demonstrated in one line using the exponential notation of complex numbers. Ironically, a long-winded 'derivation' was chosen instead because this convenient notation is 'not particularly suitable $[\ldots]$ in practice because it contains complex quantities'. It remains unclear how treating the real and imaginary parts (i.e. the cosine and sine terms) separately is more 'suitable [...] in practice'. Surprisingly, the second part of the book then assumes an intimate knowledge of complex numbers in the context of wave equations.

At times, the organization of the book is arguably confusing, with notions introduced after they have been used (Dirac $\delta$, crystallographic restriction theorem, Friedel's law, Brillouin zone boundaries, the Bloch theorem) or not introduced at all (symmetry, symmetry axis, symmetry operation, operator, polar axis) or discussed in unrelated sections (e.g. kinematic model in 'Lattice diffraction'; electron and neutron scattering are subsections in 'Anomalous dispersion'; elastic scattering in 'Waves in space'; 'The Patterson method' and 'Charge flipping' are not part of 'Solution of crystal structures'; 'Total scattering' is presented independently of 'The Patterson method'). 'Intensity calculation' could perhaps be more aptly called 'Systematic absences' or 'Reflection conditions'.

Occasionally, I got the impression that supposedly cuttingedge topics were crammed in, but were given insufficient space to be useful [the Rietveld method: 2 pages; total scattering: 2 pages; quasi-periodics: 4 pages (note that there are no diffraction patterns in a book with 'reciprocal space' in the title); disorder: 1 page (likewise with no diffraction patterns)]. To my taste, these could have been omitted or their treatment reduced to a short paragraph to save space for the fundamentals. Likewise, a lengthy derivation in the section 'Lattice dynamics' is given only to 'emphasize its complexity', which is not a rewarding insight. A similarly long-winded derivation of $C_{v}$ appears hardly related to the title of the book.

The tone of the book is ambivalent. It is generally written in an amicable style, which is very welcome. However, in the second part, the tone sometimes becomes strangely overbearing. The 'Brillouin zone' is redefined as an arbitrary 'unit cell in reciprocal space' (in crystallography unit cells are parallelepipeds, which Brillouin zones in general are not) with an aside that this is owing to the topic being 'poorly understood by many physicists and chemists'. Here, a misconception in another textbook is pointed out, which feels out of place considering the book's own level of errors. One can assume that the ambiguity of the wavevector is well understood by the target audience (solid-state physicists). Even though different 'unit cells' in $k$-space can be chosen, these do not constitute 'Brillouin zones' as generally defined. A textbook is not the place to redefine well accepted notions. Moreover, the text contradicts itself in this respect by indirectly stating that the Brillouin zone boundary is special (where 'standing waves' are located) and that everything outside the first Brillouin zone is 'unphysical' and 'imaginary'.

Writing a book aimed at newcomers is probably the most difficult task a scientific author can face. A good introductory book is concise and easily digestible without sacrificing correctness and rigour. It shows the fundamentals, but also the complexities that arise from them, as therein lies the beauty of science. Clearly, the author strived for an easy read and assumed a certain amount of prior knowledge. This is not a valid excuse for the shortcomings discussed above, which will leave beginners in a deeply confused state. The erroneous derivations punish those who wish to dig deeper. Yet, these are precisely the readers that we should attempt to attract to our science. Moreover, by painting crystallography as an imprecise and convoluted science, the book will hardly pique the interest of solid-state physicists. In truth, crystallography's multidisciplinary approach while retaining a high level of rigour is one of its main attractions.

All this is unfortunate as the book clearly has potential, as mentioned at the start of this review. Hopefully, a future thoroughly revised and proofread version will be able to bridge the gap between crystallography and solid-state physics, and present a clear and concise view of reciprocal space. 